\title{
Mass segregation in M 67 with 2MASS
}

\author{
Ch. Bonatto and E. Bica \\ Universidade Federal do Rio Grande do Sul, Instituto de Física, CP 15051, Porto Alegre 91501-970, RS, Brazil \\ Received 9 December 2002 / Accepted 10 February 2003

\begin{abstract}
We make use of the homogeneity, depth and sky coverage of the 2MASS catalogue to study spatial variations of the stellar luminosity function in the intermediate age cluster M 67 (NGC 2682). We find that the central region $\left(R<3.3^{\prime}\right)$ has a depletion of G 0 and G 5 stars with respect to the intermediate annulus $\left(3.3^{\prime}<R<7.4^{\prime}\right)$ and the halo $\left(12^{\prime}<R<24^{\prime}\right)$. In the non-eroded part of the mass function the slope is steeper in the intermediate annulus than in the central region, suggesting an enhancement of F 0 stars with respect to turnoff and A 5 stars in the latter zone. In the halo, the G 0-G 5 stars are not depleted as compared to the central region, but the overall mass function slope is rather shallow. This suggests that the halo is enriched in low mass stars, transferred there from the inner parts as a consequence of the internal dynamical evolution, but tidal losses to the Galactic field have also been important.
\end{abstract}

Key words. Galaxy: open clusters and associations: general

\section{Introduction}

Mass segregation is an expected phenomenon in open clusters that are old enough for their stellar content to be dynamically relaxed (Binney \& Tremaine 1987). Observationally, it is not an easy task to detect mass segregation since, ideally, one has to go deep photometrically to attain the cluster's low mass stars, and field stars contamination has to be properly taken into account.

A suitable object in which mass segregation can be studied is the moderately rich open cluster M 67 (NGC 2682), located at $\mathrm{J} 200008^{\mathrm{h}} 51^{\mathrm{m}} 25^{\mathrm{s}}+11^{\circ} 49^{\prime} 50^{\prime \prime}\left(\ell=215.68^{\circ}, b=31.93^{\circ}\right)$, consequently it is a high-latitude, not heavily contaminated object. Its apparent size can easily be traced visually to $R \approx 10^{\prime}$, as can be inferred from Fig. 1. The reddening value in the cluster direction as predicted by the Schlegel et al.'s (1998) dust emission maps is $E(B-V)=0.035$, which also makes M 67 suitable for deep photometry. The WEBDA database (Mermilliod 1996 - http: //obswww . unige. ch/webda) provides the following properties for $\mathrm{M} 67$ : age $=2.6 \mathrm{Gyr}, E(B-V)=0.059$, $d_{\odot}=0.91 \mathrm{kpc}$ and $[\mathrm{Fe} / \mathrm{H}]=-0.15$. However, there is no consensus so far concerning M 67's precise age, e.g. Carraro et al. (1994) provide an age based on classical isochrones of $4.3 \mathrm{Gyr}$, while with overshoot models they estimate $4.8 \mathrm{Gyr}$, both somewhat older than that in WEBDA.

Montgomery et al. (1993) presented $U, B, V$, and $I$ $\mathrm{CCD}$ photometry in a $30^{\prime} \times 30^{\prime}$ region of $\mathrm{M} 67$, reaching $M_{V}=10.55$. They found a substantial binary sequence with at least 38 percent of cluster stars as binaries. They found evidence for more massive stars of the cluster to be more centrally concentrated, along with a turnover in the cluster luminosity

Send offprint requests to: $\mathrm{Ch}$. Bonatto, e-mail: charles@if.ufrgs.br function at low masses, which may be due to dynamical relaxation of the cluster.

M67 is one of the best-studied open clusters in the optical domain, in which the phenomenon of mass segregation has been known for a long time. van den Bergh \& Sher (1960) found evidence of a turnover on the luminosity function and a decline towards lower masses. Mathieu \& Latham (1986) pointed out that binaries and blue-stragglers are more centrally concentrated with respect to single stars. Francic (1989) also detected mass segregation in M 67 based on proper-motion membership probabilities. Fan et al. (1996), sampling a large spatial area, analyzed mass segregation and the resulting effects on the luminosity function.

The relaxation time for an open cluster can be calculated as $t_{\text {relax }}=\frac{N}{8 \ln N} t_{\text {cross }}$, where $t_{\text {cross }}=r / v$ is the crossing time (Binney \& Tremaine 1987). Assuming a radius of $r=5 \mathrm{pc}$ and a typical velocity dispersion of $v \approx 1 \mathrm{~km} \mathrm{~s}^{-1}, t_{\text {relax }} \approx 90 \mathrm{Myr}$ for a cluster with $N=10^{3}$ stars, and $t_{\text {relax }} \approx 700 \mathrm{Myr}$ for a very-rich one with $N=10^{4}$ stars. Since M 67 has a present-day mass of about $800 M_{\odot}$ (Montgomery et al. 1993) and an age of 3-4 Gyr, even with a primordial mass considerably larger, it is expected to be a relaxed system with a well-defined core/halo structure.

Our motivation to undertake the present study was the fact that no infrared study (beyond $1 \mu \mathrm{m}$ ) on mass segregation of M67 has been previously made. The present approach is as well a feasibility study to explore the usefulness of the 2MASS public database for such investigations. Owing to the sky coverage which is crucial to properly take into account the stellar background contribution to the cluster, the depth and homogeneity of the Two Micron All Sky Survey (hereafter 2MASS, Skrutskie et al. 1997 - 


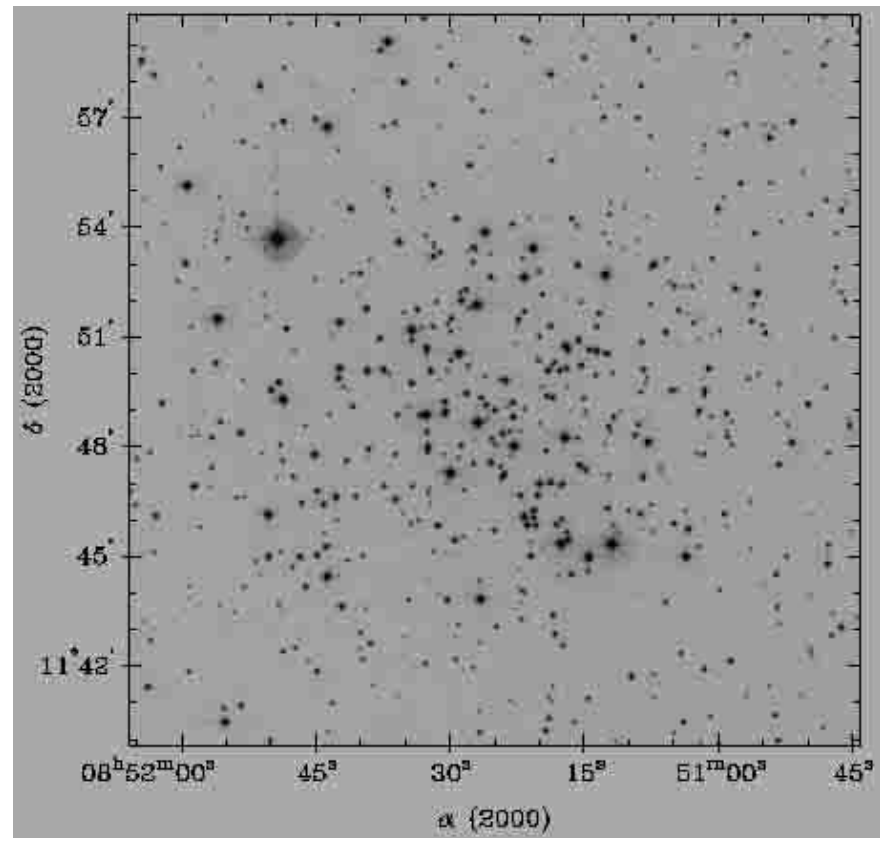

Fig. 1. $20^{\prime} \times 20^{\prime}$ XDSS B image of M 67 .

http://www.ipac. caltech.edu/2mass/releases/second/), we decided to use 2MASS photometry to probe this cluster to search for the signatures of these dynamical effects. In Sect. 2 we present the colour-magnitude diagram (CMD) and derive cluster parameters. In Sect. 3 we study the cluster structure. In Sect. 4 we discuss the luminosity functions in terms of the cluster structure. Finally, in Sect. 5 concluding remarks are given.

\section{The 2MASS photometry}

We employed in this work photometry from the 2MASS second incremental data release. Star extractions have been performed using the VizieR tool at http://vizier.u-strasbg.fr/viz-bin/VizieR?-source= 2MASS. The 2MASS photometric errors as a function of magnitude can be seen in Soares \& Bica (2002) for the three bands $J, H$ and $K_{S}$. Since M 67 is basically unreddened and the errors are more important for $K_{S}$ at a given magnitude, we decided to use $J$ and $H$ to probe the fainter stars with smaller errors.

In order to derive parameters for M 67, we use solar metallicity Padova isochrones from Girardi et al. (2000). These isochrones are computed with updated opacities and equation of state, and a moderate amount of convective overshoot with initial masses ranging from $0.15-7 M_{\odot}$.

We present in Fig. 2 the $J$ and $(J-H)$ 2MASS CMD for stars extracted inside a radius of $15^{\prime}$. The best isochrone fit is obtained for solar metallicity, an age of $3.2 \pm 0.4 \mathrm{Gyr}$ and null reddening. Accordingly, the distance modulus turns out to be $(m-M)_{J}=9.7$, corresponding to a distance of $d_{\odot} \approx$ $0.87 \pm 0.04 \mathrm{kpc}$. As reference, we indicate in Fig. 2 stellar masses and spectral types. Notice that we are reaching $M_{J} \approx 7$ which corresponds to $\mathrm{K} 0$ stars.

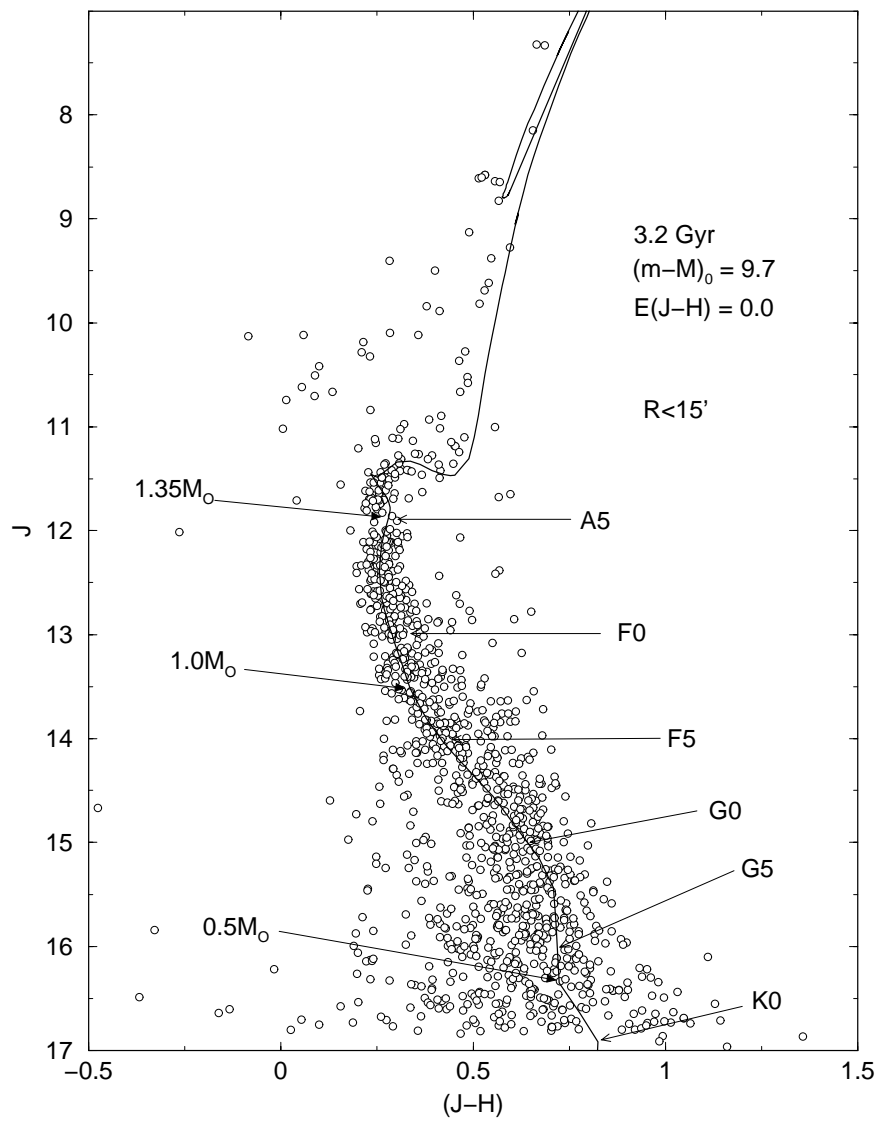

Fig. 2. Solar metallicity Padova isochrone fitting to M67; extraction is $15^{\prime}$ in radius. Spectral types and masses of MS stars are indicated.

As pointed out by Janes \& Phelps (1994), age estimates for M 67 vary from 3.4 Gyr to $6.0 \mathrm{Gyr}$. In those optical studies, the shape of the turnoff (TO) was often mismatched by the adopted isochrone. We stress that in the present infrared study, the solar metallicity Padova isochrone describes very well the TO (Fig. 2), thus constraining the age to a value around 3.2 Gyr. This age is a lower-limit to those listed in Janes \& Phelps (1994).

To estimate field star contamination we collected stars from two offset (north and south) circular fields, located at $35^{\prime}$ away in either direction from the cluster center and with extraction radius of $15^{\prime}$. The offset stars are shown in a $J \times(J-H)$ CMD (Fig. 3), in which we included the 3.2 Gyr Padova isochrone fitted to M 67, for comparison. It is apparent that features such as the main sequence (MS), TO, subgiant and giant branches, which are evident in the M67 CMD, are not present in the CMD of the offset fields. However, it is not excluded some contamination of M 67 stars, especially MS, at such distances from the main body of $\mathrm{M} 67$.

\section{Cluster structure}

M67 is a loose open cluster with a central star concentration, which is apparent in a blue Digitized Sky Survey image (Fig. 1). Due to its rather high galactic latitude, the field of M 67 is uncrowded $\left(\approx 0.73\right.$ stars $\operatorname{arcmin}^{-2}$ as extracted with 2 MASS $)$ which favours cluster structural analyses. 


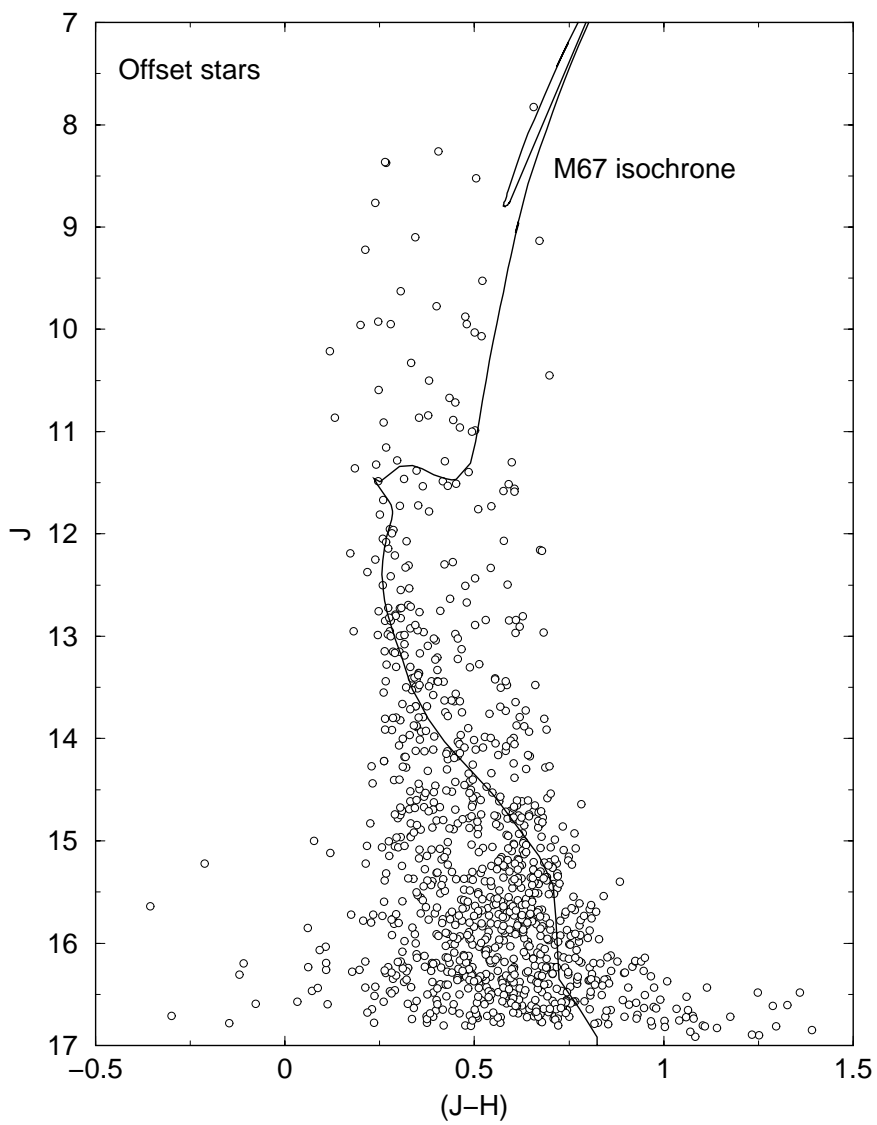

Fig. 3. CMD of the offset stars including the M67 3.2 Gyr Padova isochrone, for comparison.

Figure 4 shows the radial distribution of star density in and around the cluster. We used concentric annuli with a step of $2.0^{\prime}$ in radius. The background level was estimated as an average of the two offset (north and south) fields.

The cluster has a well-defined core extending to $R=7.4^{\prime}$ with a secondary excess in the central $3.3^{\prime}$ which we will refer to as central region. There occurs a plateau for $7.4^{\prime}<R<$ $12^{\prime}$, and after that, a halo extending up to $R \approx 24^{\prime}$. The cluster image in Fig. 1 corresponds to the higher surface-brightness region, extending up to the plateau in Fig. 4. In Table 1 we give the corresponding linear dimensions for the given cluster structures, assuming a distance $d_{\odot}=0.87 \mathrm{kpc}$.

Fan et al. (1996) found evidence of non-sphericity in M 67 based on density and definition of star sequences in CMDs around M67 in the cluster region $5.0^{\prime} \leq R \leq 16.7^{\prime}$. This structural deviation might also be a tidal extension due to the Galactic field (Bergond et al. 2001). Although such distortions might occur, we apply a King's law (1966) to derive first order structural parameters. The cluster core radius $r_{\text {core }}$ can be analitically calculated by fitting a King's surface density profile $\left.\sigma(r)=\frac{\sigma_{0}}{1+(r / r}\right)^{2}$ to the background-subtracted radial distribution of stars in M 67 (Fig. 4). The resulting fit is shown in Fig. 4 as a long-dashed line. The calculated core radius $r_{\text {core }}=4.86^{\prime}$ $(1.23 \mathrm{pc})$ lies nearly halfway between the present central region and intermediate annulus radii. The denominations central region and intermediate annulus are similar to those defined by Fan et al. (1996).

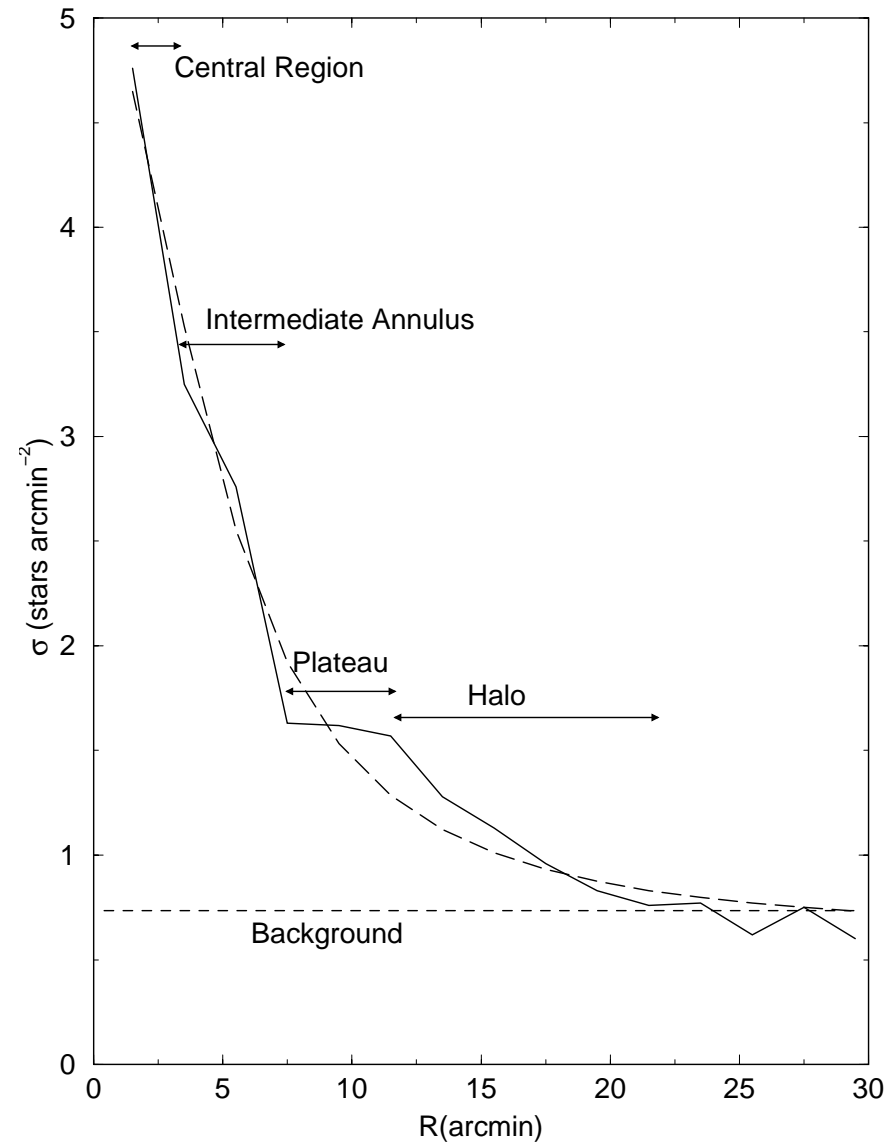

Fig. 4. Radial distribution of surface star density for M 67 (solid line). The background level is an average of 2 offset positions and is shown as a horizontal dashed line. Owing to the large number of stars in each bin the resulting Poisson errors are negligible. Surface density profile fit (long-dashed line) to the background-subtracted radial distribution of stars; the resulting core radius is $r_{\text {core }}=4.86^{\prime}$.

\section{Luminosity functions}

In the present section we study the luminosity function of each spatial zone identified in the cluster surface stellar density profile (Fig. 4). For each zone we extracted stars within the corresponding annuli, and show the results in Fig. 5. As comparison, we also include in Fig. 5 an extraction surrounding the halo $\left(20^{\prime}<R<30^{\prime}\right)$ and the offset field (average of the north and south fields at $35^{\prime}$ away, with extraction radius $R=15^{\prime}$ ). In each panel we overimpose the offset field histogram scaled to the same area as that of the respective cluster zone. By subtracting the field contribution we obtained the cluster's intrinsic luminosity function (except for completeness corrections). Taken as face values, the central region luminosity function appears to be severely depleted at low-mass stars ( $\mathrm{G} 0$ at $J=15)$. The intermediate annulus and plateau have comparable luminosity functions with little evidence of the low-mass depletion. In the halo the distribution's peak corresponds to fainter stars. The halo itself does not appear to extend further out than $R \approx 20^{\prime}$.

The results shown in Fig. 5 basically confirm Montgomery et al.'s (1993) tendency for more massive stars to be more centrally concentrated, as well as a turnover in the cluster luminosity function at low masses. 


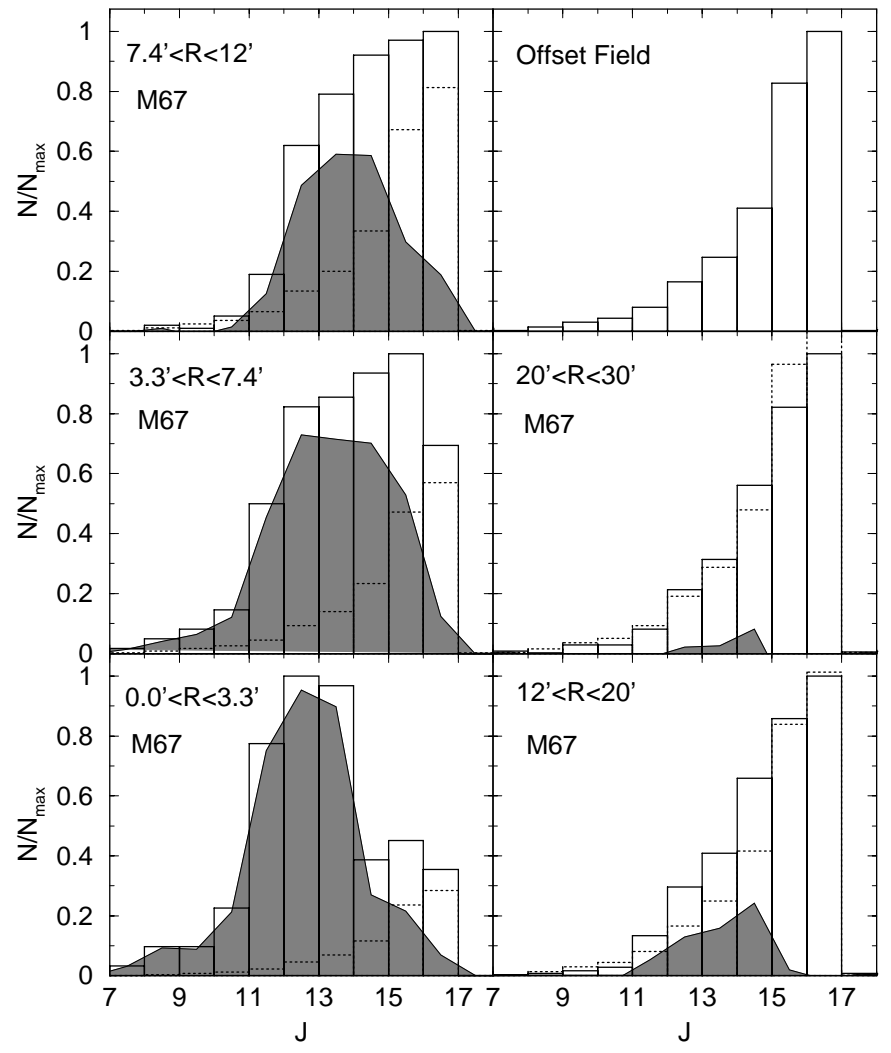

Fig. 5. Spatial distribution of luminosity functions for M67. Offset field (upper-right panel) is shown to scale in each cluster panel (dotted histogram). The hashed curve represents the subtraction of cluster and scaled field. Histograms are normalized to the bin containing the maximum number of stars.

We compare the present M67 luminosity functions with that of a non-relaxed dense open cluster at low galactic latitudes in order to infer on possible effects of completeness, especially at the fainter end of the luminosity functions. NGC 2362 is a young cluster located at $\mathrm{J} 200007^{\mathrm{h}} 18^{\mathrm{m}} 42^{\mathrm{s}}-24^{\circ} 57^{\prime} 15^{\prime \prime}$ $\left(\ell=238.18^{\circ}, b=-5.54^{\circ}\right)$. Its parameters in the WEBDA database are as follows: $d_{\odot} \approx 1.40 \mathrm{kpc}, E(B-V)=0.095$ and age $\approx 8 \mathrm{Myr}$, thus non-relaxed. We show an R XDSS image of NGC 2362 in Fig. 6 where its rather high star density is evident.

The cluster is also available in the 2MASS second incremental data release, and we made extractions as well with the VizieR tool. Cluster and offset circular fields have been extracted with a $4^{\prime}$ radius. Owing to the low galactic latitude, we extracted 4 offset fields (N, S, W and E) at $15^{\prime}$ away from the cluster center to improve star count statistics.

We show in Fig. 7 the NGC 2362 luminosity function together with the average of those of the offset fields. Both distributions peak at $J \approx 16$, and the corresponding difference at $J \approx 15$. Consequently, completeness effects do not appear to affect stars brighter than $J=15$, and consequently, the conclusions drawn above about the M67 mass functions spatial dependences appear to be intrinsic.

The fact that we detected the M67 luminosity function turnover as in e.g. Montgomery et al. (1993) and Fan et al. (1996) supports that at such faint magnitudes both infrared and

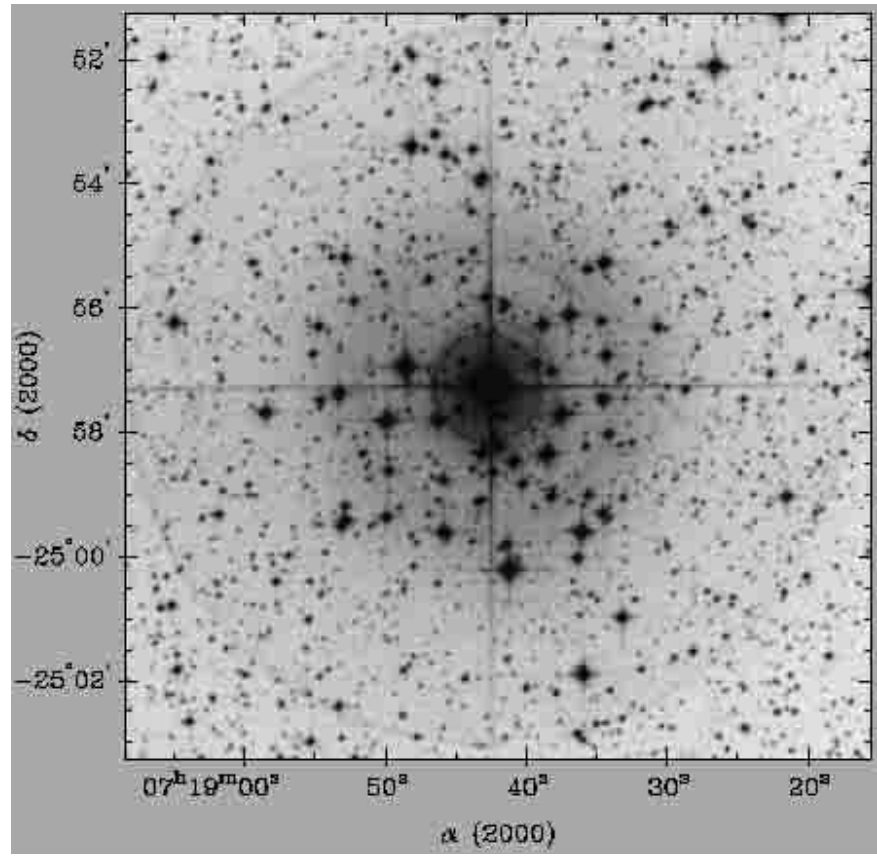

Fig. 6. $12^{\prime} \times 12^{\prime}$ XDSS $R$ image of NGC 2362 .

Table 1. M67 spatial zones.

\begin{tabular}{lcccc}
\hline \hline Zone & $\Delta R\left(^{\prime}\right)$ & $\Delta R(\mathrm{pc})$ & Fit & $x$ \\
\hline Central region & $0.0-3.3$ & $0.0-0.8$ & TO-F 0 & $2.41 \pm 0.30$ \\
Interm. annulus & $3.3-7.4$ & $0.8-1.9$ & TO-F 0 & $3.51 \pm 0.20$ \\
Plateau & $7.4-12$ & $1.9-3.0$ & TO-F 0 & $4.95 \pm 0.20$ \\
Halo & $12-24$ & $3.0-6.1$ & TO-G 0 & $2.17 \pm 0.30$ \\
\hline
\end{tabular}

Notes. Column 4: stellar types included in the luminosity function fit; Col. 5: mass function slope $N(M) \propto M^{-x}$.

optical studies are not significantly affected by completeness effects.

In order to quantify the mass segregation in M 67, we calculate the mass function slopes $(x)$ which best fit the data in the form $\frac{\mathrm{d} N}{\mathrm{~d} M} \propto M^{-(1+x)}$, taking into account the main sequence and the turnoff. For this mass range (Fig. 2), we use a mass to light ratio $L \propto M^{4}$ (e.g. Shu 1982). The results are in Table 1 for each region and mass range. Notice that in all cases the mass function slope is steeper than a Salpeter value $x=1.35$. Kroupa (1998) pointed out that binaries appear to produce steeper slopes than that of a mass function for single stars. Since M 67 is known to have a substantial fraction of binaries (Montgomery et al. 1993), part of the large slope values (Table 1) can be accounted for by binarism.

We show in Fig. 8 luminosity function fits for the background subtracted luminosity functions of the (i) central region, (ii) merged intermediate annulus and plateau and (iii) halo. The intrinsic uncertainty can be assessed from the range of slopes quoted in the figure.

Previous studies of radial distribution of stars found that blue stragglers are more centrally concentrated than other types of stars in M67 (e.g. Mathieu \& Latham 1986 and 


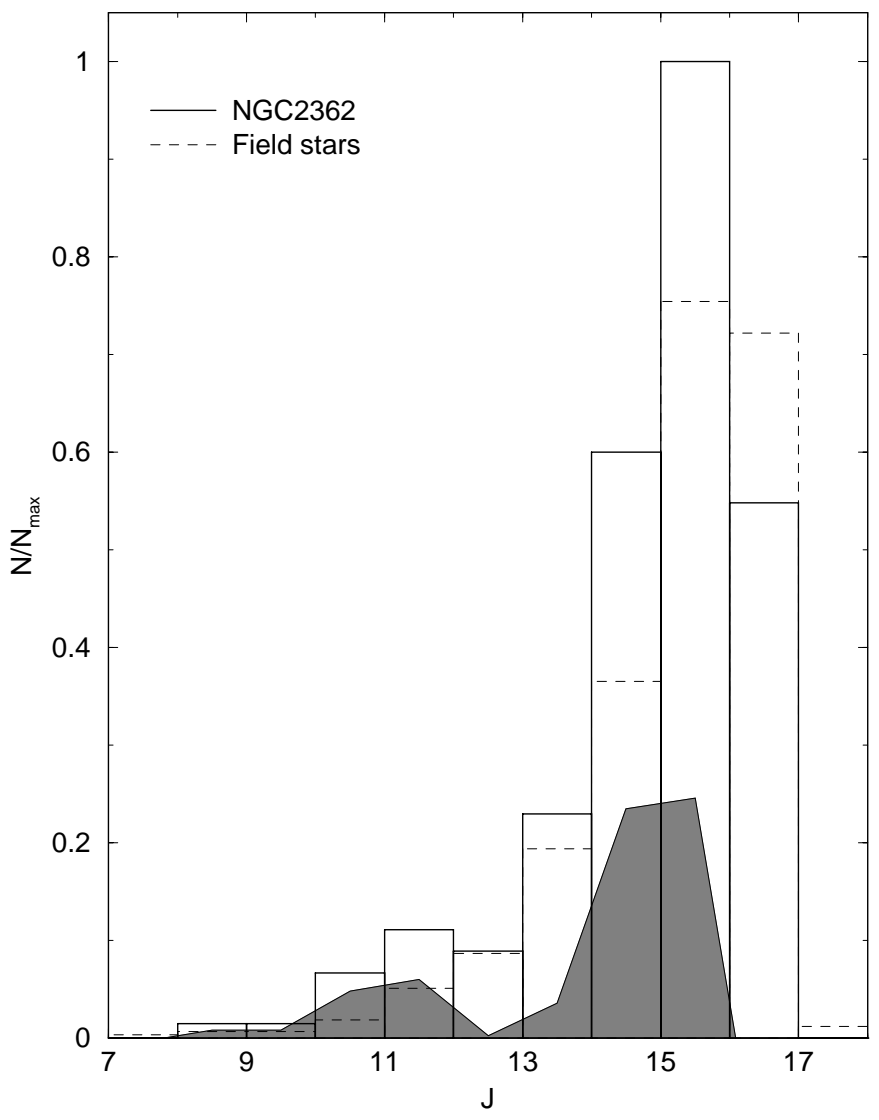

Fig. 7. Same as Fig. 5 for NGC 2362.

Fan et al. 1996). However, we stress that we restricted the luminosity function fits only to stars later than the TO (Table 1).

In the non-eroded part of the mass function the slope is steeper in the intermediate annulus than in the central region, suggesting an enhancement of F 0 stars with respect to turnoff and A 5 stars in the latter zone (Fig. 8). In this region, we find a relatively flat slope $x=2.41 \pm 0.30$ suggesting that low-mass stars have been preferentially transferred to outer regions as expected from the dynamical evolution.

For the intermediate annulus/plateau region (Fig. 8), we find the mass function with the steepest slope $(x=3.87 \pm 0.20)$, corresponding to an enhancement of low-mass stars of spectral type F 5. Probably this is related to the mechanism of star transfer throughout the cluster as a consequence of the natural dynamical evolution.

In the halo, the G0-G 5 (Fig. 8) stars are not depleted as compared to the central region, but the overall mass function slope is rather shallow $x=2.17 \pm 0.30$. This suggests that the halo is enriched in low mass stars accreted from the inner parts but that tidal losses to the Galactic field have been effective. In addition to star losses to the Galactic static field, open clusters are subjected to repeated disk shockings with the galactic Plane (Bergond et al. 2001). At the solar radius, an open cluster crosses the disk approximately 10-20 times before its dissolution (de la Fuente Marcos 1998a,b). Chupina \& Vereshchagin (1998) detected several star clumps inside the low-density extended halo of M67. Since this kind of clump is expected as

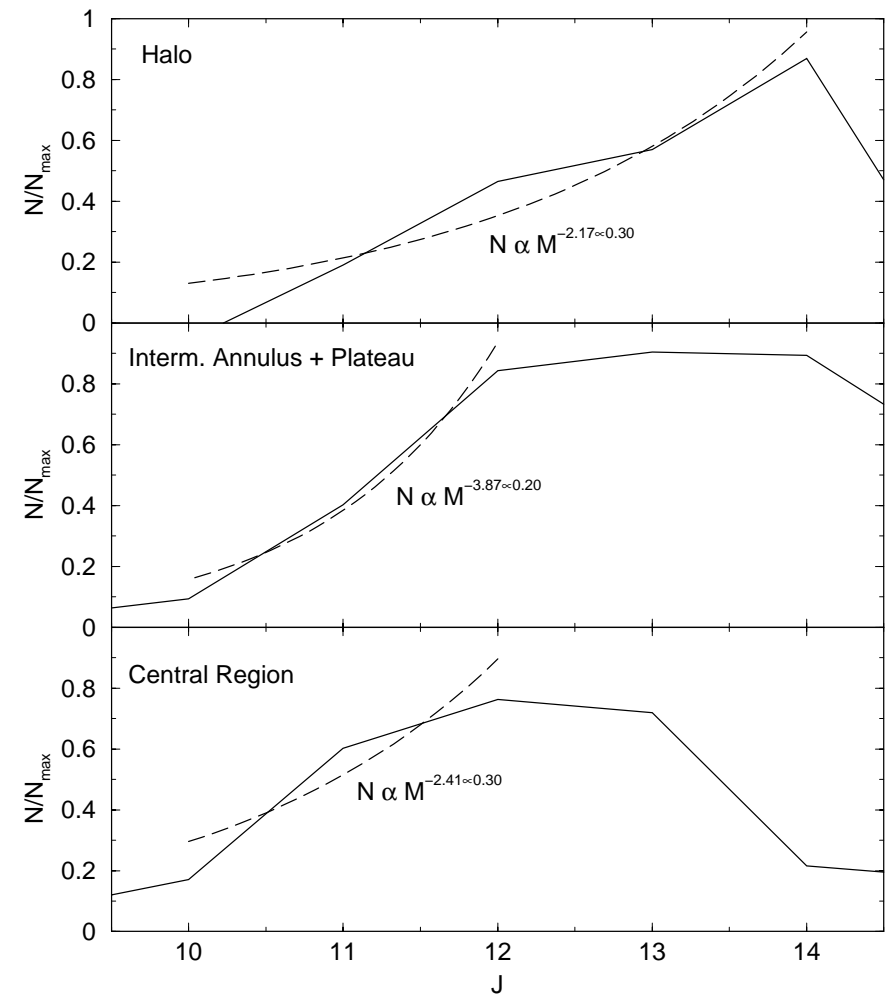

Fig. 8. $N(M) \propto M^{-x}$ (dashed line) fits to selected luminosity functions (solid line) of M 67. Note that the fits include only the stellar masses indicated in Table 1.

a consequence of disk shocking events (Bergond et al. 2001), M 67 clumps might be debris from the last disk crossing.

The presently derived luminosity function slopes are comparably high as that found for the overall stellar content of M 67 ( $x=3.49)$ by Francic (1989). With respect to Fan et al.'s (1996) radial dependence of slopes, we note that they sample different spatial regions as compared to the present study. Fan et al. (1996) find a decreasing gradient outwards, while we find first an increase followed by a decrease in the halo. In addition to the different region samplings and spectral domains, the way the background is taken into account may have produced differences. Since binarism is important in M 67 (Montgomery et al. 1993), we remind that the basic effect on the observed luminosity functions is to underestimate the relative number of low mass stars, and consequently to flatten them (Kroupa 1998). In the present case, we have assumed the luminosity functions at face values.

\section{Concluding remarks}

M 67 has been used in the literature as a probe for different dynamical aspects of an evolved open cluster. Basically, previous works employing optical data, detected mass segregation and a luminosity function turnover in the central parts. The present study for the first time employs infrared data from 2MASS. In this sense, this work is a test of the usefulness of 2MASS for such purposes, and the results we found are interesting. We confirm earlier results on mass segregation although with somewhat different properties, as well as the central luminosity 
function turnover. One of the main advantages of 2MASS is its sky coverage which allows a proper consideration of field contributions.

We study the spatial distribution of mass/luminosity functions throughout the different structural regions of this intermediate-age open cluster. Several features appear in the observed profile which do not all follow a King's law in detail. We identify these features as the (i) central region, (ii) intermediate annulus, (iii) plateau and (iv) halo. In the non-eroded part of the mass function $\left(N(M) \propto M^{-x}\right)$, we measured its slope and found that it increases from $x=2.41$ in the central region to $x=4.95$ in the plateau, decreasing again in the halo to $x=2.17$. These values must reflect the mechanisms of star transfer throughout the cluster as a consequence of the cluster's internal dynamical evolution and losses to the Galactic tidal field.

Acknowledgements. This publication makes use of data products from the Two Micron All Sky Survey, which is a joint project of the University of Massachusetts and the Infrared Processing and Analysis Center/California Institute of Technology, funded by the National Aeronautics and Space Administration and the National Science Foundation. We employed data from CDS/Simbad/VizieR (Strasbourg) and Digitized Sky Survey images from the Space Telescope Science Institute (U.S. Government grant NAG W-2166) obtained using the extraction tool from CADC (Canada). We also made use of the WEBDA open cluster database. We acknowledge support from the Brazilian Institution CNPq. We thank Leandro Kerber for interesting discussions. We thank an anonymous referee for helpful comments and suggestions.

\section{References}

Bergond, G., Leon, S., \& Guilbert, J. 2001, A\&A, 377, 462

Binney, J., \& Tremaine, S. 1987, in Galactic dynamics (Princeton, USA: Princeton University Press)

Carraro, G., Chiosi, C., Bressan, A., \& Bertelli, G. 1994, A\&AS, 103, 375

Chupina, N. V., \& Vereshchagin, S. V. 1998, A\&A, 334, 552

de la Fuente Marcos, R. 1998a, PASP, 110, 1117

de la Fuente Marcos, R. 1998b, A\&A, 333, L27

Fan, X., Burstein, D., Chen, J-S., et al. 1996, AJ, 112, 628

Francic, S. P. 1989, AJ, 98, 888

Girardi, L., Bressan, A., Bertelli, G., \& Chiosi, C. 2000, A\&AS, 141, 371

Janes, K. A., \& Phelps, R. L. 1994, AJ, 108, 1773

King, I. 1966, AJ, 71, 64

Kroupa, P. 1998, MNRAS, 298, 231

Mathieu, R. D., \& Latham, D. W. 1986, AJ, 92, 1364

Mermilliod, J. C. 1996, in The origins, evolution, and destinies of binary stars in clusters, ASP Conf. Ser., 90, 475

Montgomery, K. A., Marschall, L. A., \& Janes, K. A. 1993, AJ, 106, 181

Schlegel, D. J., Finkbeiner, D. P., \& Davis, M. 1998, ApJ, 500, 525

Shu, F. H. 1982, in The Physical Universe (Mill Valley: University Science Books), 146

Skrutskie, M., Schneider, S. E., Stiening, R., et al. 1997, in The Impact of Large Scale Near-IR Sky Surveys, ed. F. Garzon, N. Epchtein, A. Omont, B. Burton, \& P. Persi (The Netherlands: Kluwer), 210, 187

Soares, J. B., \& Bica, E. 2002, A\&A, 388, 172

van den Bergh, S., \& Sher, D. 1960, PDDO, 2, 203 